Archives

2| 1988

Varia

\title{
Urbanisation et mouvement national en Europe orientale dans la première moitié du XIX siècle
}

\section{Emil Niederhauser}

\section{(2) OpenEdition}

\section{Journals}

Édition électronique

URL : http://journals.openedition.org/ccrh/2959

DOl : $10.4000 /$ ccrh.2959

ISSN : $1760-7906$

Éditeur

Centre de recherches historiques - EHESS

Édition imprimée

Date de publication : 15 avril 1988

ISSN : 0990-9141

Référence électronique

Emil Niederhauser, «Urbanisation et mouvement national en Europe orientale dans la première moitié du XIXe siècle », Les Cahiers du Centre de Recherches Historiques [En ligne], 2 | 1988, mis en ligne le 13 avril 2009, consulté le 08 mai 2019. URL : http://journals.openedition.org/ccrh/2959; DOI : 10.4000/ ccrh.2959

Ce document a été généré automatiquement le 8 mai 2019.

Article L.111-1 du Code de la propriété intellectuelle. 


\title{
Urbanisation et mouvement national en Europe orientale dans la première moitié du XIX ${ }^{\mathrm{e}}$ siècle
}

\author{
Emil Niederhauser
}

1 Les notions de ville et d'urbanisation sont à utiliser avec précaution pour la période antérieure à la Révolution de 1848 et aux réformes de 1860. Si l'on compare la situation de l'Europe occidentale à la même époque, il n'y a guère de villes dans la première, au sens moderne du terme, à l'exception de quelques capitales.

Dans cette Europe orientale jusqu'à l'époque qui nous intéresse, on peut distinguer trois sous-régions. C'est dans la Rzecz pospolita (le Royaume polono-lithuanien incluant l'Ukraine et les pays baltiques) et dans l'Empire des Habsbourg, que se trouvent les villes dites royales d'origine médiévale; ces villes sont à peu près comparables aux villes occidentales du Moyen-Age; dotées d'une industrie artisanale et d'une activité commerciale non négligeables, elles ont décliné et sont devenues à moitié agraires dès le début $\mathrm{du} \mathrm{xVI}^{\mathrm{e}}$ siècle. En Russie, ce type de ville européenne n'existait pas; c'était le commerce et les fonctions administratives qui déterminaient le caractère des agglomérations. Dans les Balkans, c'était plus encore et quasi exclusivement, le commerce.

3 Si l'on fait l'hypothèse d'une très lente évolution économique et sociale, presque imperceptible sous les apparences féodales, que dire des facteurs susceptibles d'entrainer une urbanisation? La fonction administrative semble être le facteur le plus important. A l'exclusion de la Pologne qui n'existe plus en tant qu'État, les capitales des États, des provinces ou royaumes en Autriche, les chefs-lieux des gouvernements en Russie, des vilaiets dans les Balkans et les capitales des États naissants - y compris les chefs-lieux des comitats en Hongrie - étaient des centres quasi urbains. Ainsi Prague, Buda, Lwow, Zagreb, Vienne, Saint-Pétersbourg, Moscou, Belgrade et Athènes.

4 Le second facteur est le commerce du blé, du bois et d'autres produits agricoles qui animaient les ports maritimes de Riga et de Varna, ainsi que les ports du Danube, la voie commerciale la plus importante à cette époque, tels que Komaron, Györ, Pest en Hongrie ; 
Braïla et Galati dans les principautés danubiennes. Il existe deux cas exceptionnels sur le continent : Bucarest, tournée en partie vers le commerce du Levant et Nijni-Novgorod, au centre de la plaine russe avec sa foire annuelle rassemblant toute la production de la Russie agraire. Braïla, devenu port libre en 1836, accueillit 685 navires en 1839; en 1847, 1383 navires dont 418 battaient pavillon anglais.

L'industrie ne vient ici qu'en troisième position, non seulement en raison de la faiblesse de la production industrielle, mais surtout parce que l'industrie manufacturière est implantée dans les villages; cette situation est particulièrement nette en Russie où l'on trouve une ceinture de gouvernements centraux, peuplés de villages qu'il faut alimenter avec la production des villes devenues agricoles. Cependant, il y a déjà des villes industrielles comme Prague. La grève des indienneurs qui s'y déroula en 1844 aurait pu se produire de la même façon à Lyon ou à Londres. Lodz, en Pologne russe, devient vers le milieu du siècle, un centre de production textile, tout à fait moderne. Pest, avec ses moulins à vapeur, devient un centre important de l'industrie alimentaire. Le statut de République libre de Cracovie, fixé par le Congrès de Vienne, a favorisé son développement, beaucoup plus que son passé d'ancienne capitale et ville des artisans allemands.

On pourrait penser que les chemins de fer ont joué un rôle dans la naissance de nouveaux centres, comme en Europe occidentale. Mais les lignes ferroviaires commencent à peine à se développer. En Russie, une première ligne est construite en 1837 entre Pétersbourg et Tsarkoie-Selo, objet de divertissement plus que facteur de développement économique. Ce n'est que dans les années 1850 que l'on commence à construire la ligne reliant Moscou à Saint-Pétersbourg. La première véritable grande ligne, reliant Varsovie à Vienne, ne sert que des intérêts stratégiques.

7 Dans un certain nombre de villes de moindre importance, les artisans sont regroupés en corporations. De tradition ancienne en Europe centrale et orientale, ces institutions font leur apparition en Russie par décret du tzar Pierre-le-Grand. Dans les Balkans, les esnafs ottomans représentent aussi la volonté du pouvoir d'organiser la vie économique et de la développer. Mais la plupart des citadins sont employés dans l'agriculture, y compris les membres des corpo-rations. D'une façon générale, la population des villes n'augmente pas; la mobilité horizontale est faible. En Russie le fait est dû au régime féodal qui ne reconnait pas aux serfs le droit de changer de résidence. En Autriche, cette mobilité est légalement possible, mais ce sont des raisons économiques qui l'empêchent; il en est de même dans l'Empire Ottoman qui ne reconnait pas le servage. Ce n'est que dans la partie russe de la Pologne, où le servage n'existe plus grâce à la constitution du Duché de Varsovie édictée par Napoléon, qu'il existe de nombreux paysans sans terre qui viennent grossir la population urbaine.

Il se produit des concentrations de population dans les villes, surtout dans les capitales, à l'occasion des grands travaux du bâtiment. L'édification des palais aristocratiques de Saint-Petersbourg et de Moscou, de Pest et de Zagreb nécessite en effet une population temporaire importante, qui retourne à la campagne après l'achèvement des travaux. Le style classique des bâtiments est caractéristique des capitales quand elles ne sont pas devenues baroques, comme Prague ou Vienne.

9 La vie culturelle constitue également un facteur d'urbanisation. Les institutions culturelles les plus importantes sont les universités, fondées vers la fin du Moyen-Age (Prague ou Varsovie) ou plus récemment (Lemberg, Pest, Moscou, Saint-Pétersbourg, Kazan ou Odessa). Elles constituent des centres de vie intellectuelle et sociale qui 
deviendront redoutables pour le pouvoir des Habsbourg, pendant la Révolution de 1848. Dans cet Empire, d'autres grandes écoles, les académies de droit ou les collèges (grandes écoles protestantes qui dispensent un enseignement ouvert aux idées nouvelles et de haut niveau) surpassent généralement les universités, plus traditionnelles. Le rôle des écoles religieuses, des facultés de théologie est également important. Mais il y a également des institutions tout à fait nouvelles, telles que les musées, les sociétés littéraires et scientifiques, la presse et les éditeurs. L'homme de lettres, qui est souvent lui-même un grand seigneur ou qui se doit de trouver un mécène pour assurer sa subsistance, devient un professionnel. Les professeurs d'université, les écrivains et les poètes édifient des fortunes considérables, ainsi que les peintres en raison de l'intérêt porté au portrait par la bonne bourgeoisie.

Cette évolution facilite l'essor d'un mouvement national. Ainsi, dans cette première moitié du XIXe siècle, hormis les Ukrainiens, les Albanais, les peuples baltiques qui n'ont pas encore de mouvements nationaux, une bonne dizaine de peuples, imitant l'exemple français, sont en passe de devenir des nations au sens moderne du terme. La structure sociale de ces peuples est différente à maints égards, ainsi que le poids de leur population. On peut définir deux grands types à partir d'une différence fondamentale. Otto Bauer, le premier, a établi une distinction entre les peuples historiques et les peuples anhistoriques, la différence tenant à l'existence chez les uns d'une noblesse, d'une aristocratie qui n'existent pas chez les autres. J'utilise les termes de nation «noble » et nation « non-noble » pour marquer cette différence.

11 Les nations « nobles » sont celles qui ont leur propre classe dirigeante, nobiliaire, et sont, de ce fait, dotées d'institutions féodales : les diètes ou institutions des Ordres. La diète est une institution privée du pouvoir réel de décision qui siège auprès du gouvernement, théoriquement auprès du monarque. La diète procure un lieu de vie politique où peuvent se confronter les principes et les exigences politiques, ce qui dépasse de beaucoup les fonctions initiales de ces États généraux. La forme est encore féodale mais le contenu appartient de plus en plus au monde moderne, bourgeois. Cette situation n'existe pas en Russie qui ne dispose d'aucune institution féodale de ce type puisque la Douma des boyards a disparu. L'aristocratie y dispose néanmoins de moyens informels pour se faire entendre. Par ailleurs, la nation russe, plus précisément l'ethnie russe, est la seule qui soit aussi une nation dotée d'un État, État-nation selon le modèle français, qui ne reconnait pas, à l'exception des Polonais, l'existence des autres ethnies. Toutes les autres nations « nobles » sont intégrées à des empires multinationaux.

12 Les nations «non nobles » n'ont pas de diètes, pas d'États généraux. Il leur manque donc un lieu de confrontation politique. Il existe également d'autres différences qui privilégient les nations «nobles" par rapport aux nations «non nobles». Il semblerait que cette distinction n'ait pas sa raison d'être dans l'empire ottoman puisque toutes les ethnies-y compris les Turcs-sont dépourvues d'institutions semblables. C'est l'organisation des Eglises qui remplace d'une certaine façon les institutions politiques. L'autorité les accepte comme telles dans le système des Milliets, mais, en les acceptant, elle refuse toute distinction ethnique. Dans la première moitié du XIX ${ }^{e}$ siècle, cette difficulté disparait du fait de l'apparition des petits États-nations.

13 En ce qui concerne les mouvements nationaux, il est possible, si l'on s'en tient à un type idéal au sens de Max Weber, de distinguer deux phases : une phase culturelle et une phase politique ; parfois elles se succèdent dans le temps, mais le plus souvent elles coïncident. La phase culturelle s'ouvre par la question de la langue nationale qu'il faut rétablir et 
diffuser auprès des membres virtuels de la nation. Presqu'en même temps, on crée un passé historique pour la nation, appelé à éveiller la conscience nationale chez tous les membres de la communauté. Ici encore, la situation est plus favorable pour les nations « nobles » car ce sont les Ordres, les États généraux qui défendent la langue et qui créent le passé; ce dernier sera plus conforme à l'évolution réelle parce que la noblesse, l'aristocratie, qui est la classe dirigeante depuis des siècles, a conservé une vision plus nette de ce passé. La phase politique débute aussi par la question de la langue; elle en exige l'usage dans la vie publique; il s'ensuit rapidement la réclamation de l'autonomie territoriale et de l'usage exclusif de la langue nationale dans l'administration. C'est ce qui se passe dans les Balkans au cours de ce long XIX ${ }^{e}$ siècle.

Qu'en est-il des couches dirigeantes des mouvements nationaux? Chez les nations «nobles », c'est évidemment la noblesse et/ou l'aristocratie. C'est le cas pour les Polonais, les Russes, les Hongrois, les Croates, les Roumains des Principautés danubiennes. On peut $\mathrm{y}$ ajouter les Tchèques, l'aristocratie de Bohème ayant joué un rôle pendant la phase culturelle, avant que la bourgeoisie ne prenne la direction du mouvement pendant la phase politique. Il en fut ainsi chez les Grecs même si les Phanariotes constituaient une sorte d'aristocratie informelle.

Chez les nations «non-nobles", ce sont les intellectuels, l'intelligentsia, très peu nombreuse, qui constitue la couche dirigeante des mouvements nationaux. Les prêtres y sont encore très nombreux et l'intelligentsia laïque est à peine naissante. C'est le cas des peuples balkaniques, des Roumains de Transylvanie, des Slovènes, des Slovaques. Parfois, cette situation durera jusqu'à la première guerre mondiale. Ces intellectuels sont parfois d'origine paysanne mais - comme l'a montré dans plusieurs ouvrages l'historien tchèque Miroslav Hroch - la plupart d'entre eux sont originaires de la petite bourgeoisie urbaine. Mais ce n'est pas tant l'origine sociale que la situation réelle de ces « éveilleurs » (c'est ainsi qu'ils se nommaient eux-mêmes) qui importe.

Pour les nobles ou l'intelligentsia ecclésiastique, la ville n'est pas le lieu où doit s'exercer obligatoirement leur activité. Ferenc Kazinczy, l'un des pionniers de la littérature hongroise qui anima la vie littéraire jusqu'à sa mort en 1831, vivait dans un petit village du lointain Nord-Est de la Hongrie historique. Cependant, c'est en général la ville, une ou parfois plusieurs villes, qui deviennent les centres des mouvements nationaux. L'un des leaders du mouvement hongrois, le comte Istvan Szechenyi, déploie de grands efforts qui portèrent leurs fruits pour faire de Pest le centre économique et culturel de la Hongrie, et par là même du mouvement hongrois. La ville, qui offre des possibilités d'information et de relations constitue un pôle d'attraction si important, qu'à cette époque empreinte de ferveur nationale, même une ville étrangère peut devenir un tel centre pendant un certain temps. La première société culturelle serbe, la Matica Srpska, qui fut un modèle pour d'autres organisations semblables chez les Slaves, est fondée à Pest en 1826, ville où vivent de gros commerçants serbes. Jan Kollar, poète slovaque et personnage important du mouvement tchèque qui fut aussi à l'origine de l'idée de la mutualité slave, fut pendant presque trente ans pasteur luthérien des Slovaques de Pest.

Dans tous les cas où une ville devient le centre d'un mouvement national, on constate la présence de membres de la nation concernée. Ainsi, Prague, centre du mouvement tchèque est habitée en majorité par des Allemands ; pourtant, à partir des années 1820, son rôle prépondérant dans le mouvement national tchèque est incontestable. Pour les Polonais, c'est Cracovie qui joue ce rôle, relayée par Paris après la grande émigration de 1831. Pour le mouvement hongrois, c'est Pest, ville multinationale habitée en majorité 
par des Allemands, qui devient le centre à cause de son importance économique et de la présence à Buda, sur l'autre rive du Danube, du centre administratif du royaume; notons que ce n'est pas Presbourg/Bratislava, où siègent pourtant les diètes et qui est le centre de la vie politique. Cette ville devient un centre slovaque grâce à la présence du seul lycée qui dispense un enseignement en langue tchécoslovaque. Zagreb, siège des diètes et centre administratif sera le centre naturel du mouvement croate. Pour les Serbes, Belgrade est l'équivalent de Zagreb, qui se développe comme centre politique et devient de ce fait un centre économique. Pour les Roumains de Transylvanie, ce sont de petites villes du pays qui jouent ce rôle, voire même Pest, où travaillent pendant des années les représentants du mouvement culturel. Bucarest l'emporte après 1821 et la fin de l'époque phanariote. Pour les Bulgares, c'est une ville étrangère, Constantinople, qui devient le centre du mouvement national quand celui-ci dépasse la phase culturelle surtout liée aux monastères, et c'est Bucarest et Braïla qui font fonction de centre du mouvement révolutionnaire au milieu du siècle. Les Slovènes sont les plus mal lotis car leur élite intellectuelle réside à Laibach/Ljubljana ou à Vienne, dans un environnement presque entièrement allemand. Pour les peuples balkaniques, la diaspora implantée dans de grandes villes commerciales joue le rôle le plus important dans la phase culturelle du mouvement; cela est surtout valable pour les Grecs : ce n'est que dans les années 1830 qu'Athènes devient véritablement un centre politique. La puissance économique appartient toujours à la diaspora, implantée en partie sur le territoire ottoman. Ainsi, les mouvements nationaux promus par des individus qui résident bien souvent dans des villages ou de petites villes obscures, finissent par se fixer dans les villes.

Je suis parti de la constatation que le facteur le plus important de l'urbanisation était la situation administrative ou politique de la ville. Nombre de villes citées sont devenues capitales d'états plus ou moins indépendants. Le seconde moitié du siècle est la véritable époque d'une urbanisation due essentiellement à des raisons économiques. Cependant, même pendant cette phase du développement capitaliste, le facteur national ne perd pas toute son importance. Une Europe orientale sous-développée connaîtra des nationalismes excessifs dont l'héritage est toujours vivant.

Dernier point: peut-on parler à cette époque d'un mouvement national russe? L'historiographie russe ou étrangère ne mentionne jamais les idées de renaissance ou de réveil national, pourtant évoquées à propos de la plupart des nations d'Europe orientale. La Russie était le seul État-nation. Ailleurs, les mouvements nationaux pouvaient se dresser contre un pouvoir central a-national ou étranger. En Russie, c'était impossible. Le pouvoir, le gouvernement s'y montrait national, surtout depuis 1812, pour que soit reconnue sa légitimité et il ne manquait jamais de mettre l'accent sur son fondement: l'orthodoxie. Les mouvements d'opposition ne pouvaient pas lutter pour l'indépendance nationale. Ils devaient se focaliser sur des questions sociales et économiques, qui n'étaient pas centrales dans les mouvements nationaux. La conséquence en fut un libéralisme timide et l'émergence d'un mouvement qui voulait éviter les maux du capitalisme et accéder au paradis du socialisme. Ce messianisme russe débordant est, à mon avis, une sorte de nationalisme, qui se cache derrière une façade de moralisme et d'universalisme. Ce n'est assurément pas le franc nationalisme des autres peuples d'Europe orientale. L'historiographie soviétique s'est pendant longtemps contenté de ce messianisme russe, précurseur du système soviétique. Ce n'est que depuis ces dix dernières années que l'on s'intéresse aux slavophiles, mouvement autrefois simplement qualifié de réactionnaire. 
20 Soulignant la situation unique de la Russie, du peuple russe, les slavophiles prônaient un certain progrès social (l'abolition du servage, par exemple). Contre le soi-disant cosmopolitisme des libéraux et des radicaux, et contre le despotisme du pouvoir, ils prônaient le retour au caractère original russe, détruit par l'européanisation forcée, imposée au peuple. C'est donc une idéologie, un mouvement que l'on serait tenté de considérer comme une sorte de renaissance nationale. Mais les slavophiles, éblouis par les vertus du simple paysan russe, n'étaient pas partisans d'une évolution capitaliste au sens occidental du terme. Leur populisme, teinté d'un peu d'anticapitalisme, trouvera un prolongement dans les mouvements populistes $\mathrm{du} \mathrm{xx}^{\mathrm{e}}$ siècle, qui n'ont pas connu la slavophilie. 